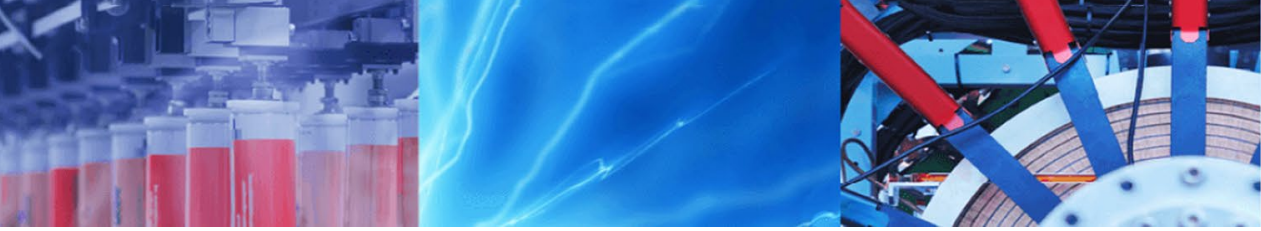

Research Article

\title{
Development of antimicrobial composite coatings for drug release in dental, orthopaedic and neural prostheses applications
}

\author{
Innocent J. Macha ${ }^{1} \cdot$ Ipek Karacan $^{2} \cdot$ Besim Ben-Nissan ${ }^{2} \cdot$ Sophie Cazalbou ${ }^{3} \cdot$ Wolfgang H. Müller $^{4}$
}

(c) Springer Nature Switzerland AG 2018

\begin{abstract}
While one of the major clinical and scientific challenges in the management of implant-related infections and postoperative complications after surgery is the application of new techniques, a new approach is pertinent in the design of medical implants to reduce bacterial infections. We have designed and tested antibiotic-containing biocomposite thin films of polylactic acid (PLA), and coralline-derived hydroxyapatite (HAp) as controlled drug delivery systems for the treatment of dental, orthopaedic and neural implant-related post-operative infections. These films can be applied to complicated designs of dental, miniaturized neural devices, cochlear or total hip replacement (THR) implants by spray or dip-coating techniques. Current results reveal that the devices could release antibiotic in a controlled manner to prevent significantly bacterial growth and biofilm production. Hydroxyapatite within the composites controls the release rate and also supplies minerals, such as calcium $\mathrm{Ca}^{2+}$ and phosphate $\mathrm{PO}_{4}{ }^{2-}$ ions, which are essential minerals for bone tissue regeneration. It is concluded from the physical, mechanical and biological properties that these coatings and devices could easily be utilized in a wide range of biomedical applications.
\end{abstract}

Keywords Implant coating · Dental implant · Drug release · Antibiotic · Hydroxyapatite · Polymer composite · Biofilm . Post-operative infection $\cdot$ Cochlear devices $\cdot$ Neural devices

\section{Introduction}

Controlled slow drug delivery systems and devices have been developed in human health-care areas in order to solve, among many, post-operative complications such as implant-related infections. Although numbers of new novel drug therapies have been developed, the main problem has been to keep the ideal concentration of drug constantly and steady during long-term delivery, which may cause medical problems. Indeed, this kind of longterm systemic drug therapies might result in toxicity in certain internal organs, such as kidneys.
In order to control drug release rate into the desired part of the human body, controlled drug delivery techniques have been used with the application of various biocomposite devices and materials, which may contain natural or synthetic biodegradable polymers or bioceramics as the matrix. In addition, a number of different drug carriers have been introduced, such as polysaccharides and calcium phosphates containing different pharmaceuticals for different treatments according to the design of the drug delivery system. It has been reported that this kind of controlled release systems provides efficacy, reduces toxicity and also improves early patient recovery $[1,2]$.

$\triangle$ Innocent J. Macha, imacha@udsm.ac.tz; Ipek Karacan, Ipek.Karacan@student.uts.edu.au; Besim Ben-Nissan, Besim.Ben-Nissan@ uts.edu.au; Sophie Cazalbou, sophie.cazalbou@univ-tlse3.fr; Wolfgang H. Müller, whmueller1000@gmail.com | ${ }^{1}$ Department of Mechanical and Industrial Engineering, College of Engineering and Technology, University of Dar es Salaam, P.O Box 35131, Dar es Salaam, Tanzania. ${ }^{2}$ School of Life Sciences, University of Technology Sydney, P.O. Box 123, Broadway, NSW 2007, Australia. ${ }^{3}$ CIRIMAT Carnot Institute, UPS-INPT-CNRS, UMR 5085, University of Toulouse, Toulouse, France. ${ }^{4}$ Mechanical Engineering and Transport Systems, Institute of Mechanics, Chair of Continuum Mechanics and Constitutive Theory, Sekr. MS 2, Einsteinufer 5, 10587 Berlin, Germany.

SN Applied Sciences (2019) 1:68 | https://doi.org/10.1007/s42452-018-0064-1 
A wide range of materials from natural materials to polymers and ceramics has been successfully used as a drug carrier for the delivery of different clinical active agents. However, marine shells and skeletons as calcium carbonate or calcium phosphates show a better promise due to their excellent biocompatibility and their unique architecture of interconnected pores, which allow loading of drugs and slow release. As stated earlier, these calcium carbonate structures can easily be fully converted into resorbable calcium phosphates with controllable dissolution rates [3-6]. The uniform interconnected porosity in these marine materials together with their controllable dissolution rate makes a complementary potential for tunable drug loading and release rates and the improvement of drug bioavailability and efficacy.

Biodegradable polymers have been widely used in medical technology [7]. Polylactic acid (PLA), polyglycolide (PGA) and their copolymers are acid anhydrides and among the widely used biodegradable synthetic polymers. They have demonstrated to be excellent biodegradable materials with non-toxic degradable products [8]. In addition, Lou and his colleagues [9] showed that the properties of synthetic aliphatic biopolymers could be tuned for specific applications. While PLA has been used in a number of clinical applications, such as sutures, stents, and dialysis media, it is also highly useful for controlled drug delivery systems in form of a matrix, because of its bio-degradation kinetics and mechanical properties $[2,10,11]$.

We have previously reported that the combination of marine bioceramics and an appropriate biodegradable polymer can form a biocomposites that have added advantages in slow drug delivery systems specifically the controlled delivery rates and the additional mineral delivery that can help both hard and soft tissue growth. It is also reported that these biocomposites display improved properties of strength and toughness [12]. Polymer-bioceramic thin-film biocomposites are flexible enough to conform to any shape and size and could be used as implant coatings, especially in dental and orthopaedic areas, and new neural devices and sensors. The most of the current orthopaedic implantable devices are produced by metallic substrates, such as Ti and CoCrMo alloys. Although metallic implants have the appropriate mechanical properties and relatively good corrosion resistance, they can cause metal-allergic reactions in certain patients and possible infection after surgery. Therefore, various types of surface modifications on the metallic implants have been designed and developed in order to eliminate these uncertainties $[13,14]$.

For this reason, antibiotic-containing PLA/hydroxyapatite ceramic thin-film biocomposites as coating materials have been developed in order to improve the surface properties of the implants, to prevent implant-related infections, and also to provide treatments for various postoperation complications, such as osteomyelitis [15].

The development of bone tissue engineering in the past has been related directly to modifications in the types of materials used. The repair process is somewhat compromised in numerous pathological and clinical situations despite bone's capability for regenerative growth. At present, a number of synthetic bone graft biomaterials, such as calcium phosphate, are available as options to an autogenous bone for augmentation, repair or substitution. New advancements associated with calcium phosphate scaffolds, such as improvements in microstructure and surface properties, have created new opportunities for bone regenerative technologies. These recent developments also make the calcium phosphate scaffolds to be thought of as biologically instructive instead of being osteoconductive scaffolds. Evidence has also suggested that certain engineering bioceramics, such as calcium phosphate, have a direct influence on the proliferation and differentiation of human mesenchymal stem cells in addition to being osteoinductive. Tissue engineering along with new bioactive molecules enhanced the possible applications of calcium phosphate as scaffolds are able to guide the behaviour of these cells and the efficiency of bone regeneration as well as being carriers of these cells.

It is widely accepted in the medical community that wound contamination as well as post-operative infections following implantation or during surgical intervention in orthopaedics and maxillofacial surgery can result in serious clinical problems and could jeopardize the osseointegration process. For these reasons antibiotics either administered orally or intravenously are often provided as prophylactics. By far the most frequent complications related to the use of implantable medical devices, such as orthopaedic or dental prostheses and endotracheal tubes, are bacterial infections. Pseudomonas aeruginosa is considered to be responsible for internal device-related infections leading to morbidity and mortality. Staphylococcus aureus, on the other hand, cause serious infections relating to severe sepsis and deep-seated infection and septic-thrombosis infections. S. aureus and Staphylococcus epidermidis strains, including methicillin-resistant S. aureus (MRSA), are the main cause of biofilm formation on the medical devices which requires surgical intervention for the treatment. Fighting biofilm formation requires deep and comprehensive understanding of the molecular bases of its formation. Osteoarticular infection is frequently caused by coagulase-negative Staphylococci as main aetiologic agents in late infections as well as Streptococci, Enterococci and anaerobes. The number of infections has been estimated to be around $0.5-2.0 \%$ of all cases, and it increases continuously due to the rise in the need for implants. 
The European Center for Disease Prevention and Control (ECDPAC) reported that approximately 4100,000 patients are estimated to acquire infections in the European Union every year. It has been estimated that at least 37,000 direct deaths occur as the result of these infection and it contributes to an additional 110,000 deaths each year. Furthermore, it has been estimated that $5 \%$ of patients undergoing surgical procedures and up to $20 \%$ of patients having intra-abdominal surgical procedures develop a surgical site infection which result in excessive hospital days and more than US\$1.6 billion hospital costs per year. Number of mitigations has been suggested including preventing and/or controlling bacterial infections. Specifically, modification or development of thin and flexible films or nanocoated multilayer devices with surface properties that have an effect on microbial adhesion or viability seems to be a promising approach for the prevention of device-related infections. On the other end, the possibility to modify the surface of medical devices biologically, chemically or physically to render the surface free of microbial adhesion is suggested to be alternative strategies as well. Multifunctional thin biocomposite films or nanocoatings can facilitate this new approach. As stated earlier for orthopaedic-dental and tissue regeneration, polymer-bioceramic biocomposites have advantages of improved bioactivity, drug encapsulation efficiency and stability as well as delivery of essential mineral ions of calcium and phosphate for bone regeneration and repair. In neural sensors, they show improved biocompatibility and allow the delivery of different drugs locally. In the past a number of drug delivery techniques for the effective treatment of neurological disorders, such as direct injection, ultrasound [16], pharmacological and physiological (noninvasive) [17], neurosurgical strategies and intracerebral implants have not been proved to be fully effective or ideal [18].

A report from the World Health Organization (WHO) shows that diseases related to the central nervous system affect nearly a billion people globally, causing immense clinical and economic burden [19]. The reports also showed that nearly $17 \%$ of the world's population suffers from different neurological disorders such as neuro-infections, multiple sclerosis, Parkinson diseases, brain injuries and strokes. There are over 600 neurological disorders, each presenting with different symptoms and affecting different parts of the central nervous system (CNS). There have been tremendous efforts towards improving diagnostic procedures and development of improved therapies for CNS. Effective and low-cost treatment for most of the CNS diseases is available; nevertheless, the treatment is limited by the blood-brain barrier (BBB) which hinders adequate penetration of therapeutic agents [20]. One of the main functions of $\mathrm{BBB}$ is to provide physical protection to the brain from getting infections; on the other hand, it acts as a physical barrier inhibiting the passage of active clinical agents to the CNS. Traditional administrations of drugs, such as oral or intravenous, are normally given in large doses to ensure some drug reaches the brain. Since this is not really targeted, it potentially poses unwanted side effects, especially in young children. The size and the type of the local delivery system are important. Nanoparticles have been designed to deliver therapeutic agents for the treatment of Alzheimer's disease. The particles could release the drug in a controlled manner for up to few days to weeks $[21,22]$. One of the drawbacks of nanocarriers is that they cannot uptake enough amount of drug to sustain a prolonged period of drug release. However, the use of biocompatible nanoparticles could increase the chance of improving the penetration of active clinical agents through BBB [23]. With the available technology and research efforts, proper design using appropriate materials could improve the delivery of drugs over a prolonged period of time. Moreover, an in-depth understanding of nanoparticles and their possible side effects are to be explored for their full potentials.

Once the above-mentioned new-generation biocomposite thin films are applied to appropriate miniaturised sensors and devices; it is envisaged that the drugs can become slowly active clinical agents delivered to a targeted area minimizing the resistance from $B B B$ with relatively smaller doses and lesser side effects compared to oral administration or systemic delivery of medication. The biocomposites consisting of hydroxyapatite derived from marine structures are developed and characterized as antibiotic delivery devices with the possible use in the treatment of dental, orthopaedic and CNS-related diseases and neural prostheses-related infections. These implant-related infections can be prevented by the use of targeted antibiotics, and this paper provides the research conducted by using this novel drug delivery system which consists of antibiotic-containing calcium phosphate microspheres and biodegradable polylactic acid (PLA) polymer biocomposite.

\section{Materials and methods}

\subsection{Materials}

Coralline materials were obtained from Queensland Australia. Gentamicin (antibiotic), sodium hypochlorite and diammonium hydrogen phosphate $\left(\left(\mathrm{NH}_{4}\right)_{2} \mathrm{HPO}_{4}, 98 \%\right)$ were purchased from Sigma-Aldrich, Castle Hill, Australia. High-purity Ti-Al-V discs were obtained from the Good Fellows UK. Bacteria Staphylococcus aureus (SH1000) for minimum inhibition concentration study, derived from 
sepsis isolate (NCTC 8325), Staphylococcus aureus (ATC 25,923 ) and Pseudomonas aeruginosa (ATCC 15692) for biofilm studies were supplied by Sigma-Aldrich, Australia.

\subsection{Methods}

\subsubsection{Hydroxyapatite from coralline materials}

According to the methods described in Ben-Nissan et al. $[24,25]$, coralline powder samples were converted into hydroxyapatite by a hydrothermal method in a Parr reactor for three hours. Initially, coralline materials had to be cleaned with $2 \%(\mathrm{v} / \mathrm{v}) \mathrm{NaClO}$, then ball-milled to obtain a fine powder of approximately $<100 \mu \mathrm{m}$ and then washed again and dried in an oven at $100^{\circ} \mathrm{C}$ for $2 \mathrm{~h}$ before use.

\subsubsection{Drug loading and thin-film composites}

Drug loading to hydroxyapatite particles was conducted in a vacuum controlled rotavapor by mixing the particles with drug solution to give $10 \% \mathrm{w} / \mathrm{w}$ loading of the drug. Since the HAp particle has nano- and mesopores, drug solution penetrates into these pores with the help of vacuum and also the particles are coated with a drug. After loading, the particles were dried in a desiccator under vacuum for further use. Film composites were developed by solution-casting method where PLA was first dissolved in chloroform and then mixed with particles loaded with drug to make PLA composites under magnetic stirrer until the uniform mixture followed by ultrasound and then casted on a Petri dish and allowed to dry under vacuum for three days. The following designation was assigned to samples as follows: PLA neat film as PLA, PLA films loaded with gentamicin (GM) as PLAGM, PLA composites with hydroxyapatite as PLAHAp, PLA composite films of hydroxyapatite loaded with gentamicin as PLAHApGM. Drug purity and drug presence in the PLA matrix were evaluated by Fourier transform infrared spectroscopy (FTIR).

\subsubsection{Dip-coating method for the implants}

Dip-coating method was used in this study in order to apply PLA biocomposites on the Ti6Al4 V flat discs and screw-shaped dental implants as a coating material. Firstly, the substrate of a Ti6Al4 V dental implant was cleaned with acetone by the ultra-sonication method. The sample was dipped into the PLA biocomposite-chloroform solution at a constant speed. This method was chosen to coat metallic substrates because of its easy applicability to complex structures.

\subsubsection{Morphology of samples}

The morphology of Gentamicin and HAp particles before and after drug loading was analysed by a scanning electron microscope (SEM) (ZEISS Supra55VP, Zeiss and Germany). The samples were fixed by conductive adhesive tape on aluminium stubs and then coated with an ultrathin coating of carbon deposited on the sample by low-vacuum sputter coating. SEM images were carefully taken at various magnifications at acceleration voltages of $5-20 \mathrm{kV}$.

\subsubsection{Fourier Transform Infrared Spectroscopy (FTIR)}

The FTIR spectra of the samples were collected from Nicolet, Magna-IR 6700 Spectrometer FTIR (Thermo Fisher Scientific, Madison USA). The samples were ground, mixed with $\mathrm{KBr}$ at a ratio of 1:100 and then pressed into transparent pellets. Transparent pellets were prepared in stainless steel die by applying a uniaxial load of $6.89 \mathrm{MPa}$ pressure (Carver press). The FTIR spectra were collected in the range $4000-400 \mathrm{~cm}^{-1}$.

\subsubsection{In vitro release experiment}

The release study was conducted in a buffer solution (phosphate buffered saline (PBS), $0.1 \mathrm{M}, \mathrm{pH} 7.4$ ) at physiological temperature conditions of $37 \pm 0.2^{\circ} \mathrm{C}$ at $100 \mathrm{rpm}$ in a temperature controlled water bath shaker. Samples with a total of $7.5 \mathrm{mg}$ drug were put in a falcon tube with a buffer solution at a volume enough to provide complete dissolution of the drug from the samples, and the volume was maintained at all times. Experiments were done in triplicates. Drug concentrations in buffer solutions were determined by UV-vis spectrophotometer (Agilent Technologies, Australia). Since Gentamicin does not absorb light in the visible spectrum; the samples were mixed with ortho-phataldialdehyde reagent prepared according to the method reported in Aviv et al. and Chou et al. [26, 27]. The absorbance corresponding to the gentamicin-reagent complex compound was then measured at $\lambda_{\max }=332 \mathrm{~nm}$. The fractional release of gentamicin was calculated with the help of a standard curve.

\subsubsection{Preparation of media for biofilm study}

Media for Pseudomonas aeruginosa ( $P$. aeruginosa) and Staphylococcus aureus (S. aureus) were prepared by dissolving $22 \mathrm{~g}$ of Mueller-Hinton II BR and $30 \mathrm{~g}$ of Trytpic Soy Broth in 1 litre of MilliQ water (18 M $)$, respectively. Then the solutions were sterilized in an autoclave at 
$121^{\circ} \mathrm{C}$ and finally stored at room temperature for further use.

\subsubsection{Bacteria strains and static biofilm formation assay}

Bacteria strains for biofilm study were cultured under aerobic and anaerobic conditions for $P$. aeruginos $a$ and $S$. aureus, respectively, at $37^{\circ} \mathrm{C}$ in a shaking incubator running at $250 \mathrm{rpm}$. Bacteria cells were grown overnight in broth media, then diluted in 1:100 and used for biofilm formation studies. Film composites samples were glued on coupons in triplicate and placed into a 12-well plate, then inoculated with bacteria cells suspension and sealed with a breathable film, and the bacteria were allowed to grow under static conditions at $37^{\circ} \mathrm{C}, 5 \% \mathrm{CO}_{2}$ for $24 \mathrm{~h}$ and $120 \mathrm{~h}$, respectively. After the experiments, the biofilm samples were stained with green fluorescent nucleic acid stain and then fixed with $4 \%$ formaldehyde. The samples were stored in the refrigerator for image analysis.

\subsubsection{Morphological study of biofilms}

Images of biofilms on the surface of the film composites were analysed using confocal laser scanning microscopy (CLSM) (Nikon A1, Tokyo, Japan). A total of eight images per specimen were randomly taken using an oil-immersion lens (60 Objective lens, aperture of 1.4) in $1.0 \mu \mathrm{m}$ slices with NIS Elements Confocal software.

\subsubsection{Biofilm topological analysis by COMSTAT}

The images recorded by CLSM were reconstructed using IMARIS, and the topology of biofilms was analysed in computer statistics software, COMSTAT, based on the methods described in Heydorn et al. study [28] and then presented as three-dimensional structures [29]. Briefly, the reconstructed tagged image format file (.TIFF) images were converted from greyscale into a black-and-white picture that could be analysed by the COMSTAT program. The threshold settings were achieved by comparing the original greyscale picture with the black-and-white picture, and the best value was chosen to give the most accurate conversion of the greyscale to the black-and-white picture. This threshold value is fixed and then used for all image stacks [30].

\subsubsection{In vitro drug release: theoretical perspective}

It is worth mentioning that the release mechanism from these devices is a complex phenomenon influenced by a number of factors. It is believed that methods of encapsulation, device dimensions and geometry, the conditions of drug release medium, type of device materials and drug-material interactions affect the release mechanism [31]. The assumption taken into consideration here is that the drug particles or particles loaded with drugs are randomly dispersed throughout the uniform matrix with known geometry and have a constant probability of finding drug at all positions in the polymer matrix [32]. Based on our previous studies, it was shown that release behaviour of a clinical active substance from degradable material follows the common pattern consisting of three stages $[33,34]$. However, a number of other studies have indicated that the behaviour of drug release from biodegradable materials can follow two or four stages [31].

The common three stages of drug release of the GM containing polymeric matrix could briefly be elucidated as follows:

1. Stage / At time zero, a polymer matrix quickly gets hydrated with buffer solution; this happens for the bulk eroding type of matrices. In this stage drugs close to the matrix surface or on the surface diffuses into the buffer, the phenomenon typically assimilated as "the initial burst". Its magnitude depends on the amount of drugs present adjacent to the matrix and the drug solubility.

2. Stage /l After the initial release, the polymer matrix starts to degrade producing soluble degradation products such as oligomers, increasing chain mobility and consequently pores formation on the polymer matrix. Degradation starts in the amorphous region of the polymer matrix leaving behind pores facilitating fluid transport, essentially for drug release [35]. The drug release by diffusion through these pores at this stage seems to be dominating the release mechanisms and mass transfer process.

3. Stage III Cumulatively, pores grow bigger as they coalesce as the degradation of the polymer matrix progresses resulting from water or PBS penetration into the polymer network and hydrolyzing the polymer into more soluble oligomers. This provides the channels for the drug diffusion towards the surface of a polymer matrix that would otherwise be too dense to allow their passage [34]. The drug is released progressively until complete polymer degradation, a process that depends on the polymer degradation behaviour.

For four-stage drug release behaviour, it has been proposed to have a secondary burst before the final stage of drug release from the biodegradable matrix. These stages were for the pure polymeric matrix, and addition of the particulate matter as drug carrier introduces additional stages to the process. The above stages do not take the dissociation of the hydroxyapatite particles 
and related release, which is related to the microspherecontaining composites, into account.

During the second and third stages, the coralline hydroxyapatite particles release the drugs that incorporated its nano- and mesopores. After the initial dissolution of the drugs in the nano- and mesopores, the structure of hydroxyapatite or calcium phosphate degrades, and more particles are generated and dissolved, hence further drug release. This introduces the drugs and the dissolved $\mathrm{Ca}$ and phosphate ions to the environment. Dissolution and diffusion processes and kinetics at this stage further influence the drug release rate.

\section{Results and discussions}

The confirmation of the physical presence of drug on the loaded hydroxyapatite particles was obtained by scanning electron microscopy (SEM). FTIR was used to evaluate an alteration of drug loaded in a PLA matrix. Figure 1 shows images of gentamicin powder (Fig. 1a), showing spherical nano- and microparticles; Fig. 1b, c shows the hydroxyapatite particles before and after loaded with gentamicin. The loaded particles display smooth surface evidence of the drug presence on their surfaces. Since the particles have meso- and nanopores, it is believed that the drug penetrated into these pores as well. The particles before loading reveal rough surfaces with platelet morphology.

Figure 2 shows FTIR shifts of gentamicin in PLA matrix consistent with IR shifts of the drug before embedded into the matrix. PLA is represented by the following peaks: $-\mathrm{C}=\mathrm{O}\left(1746 \mathrm{~cm}^{-1}\right),-\mathrm{C}-\mathrm{H}\left(1451-1358 \mathrm{~cm}^{-1}\right),-\mathrm{C}-\mathrm{O}$ ester $\left(1180-1043 \mathrm{~cm}^{-1}\right)$ and $-\mathrm{C}-\mathrm{O}$ stretch $\left(867-751 \mathrm{~cm}^{-1}\right)$. It is suggested that there is no evidence of chemical interaction between the drug (GM) and the polymer or chloroform. The drug is not regenerated or consumed in any reaction within the polymer matrix. As shown in $2 \mathrm{~B}$ gentamicin was detected in the polymer matrix with IR shifts as minor peaks, amide I-II groups, which are $\mathrm{N}-\mathrm{H}$, stretch (1619 and $1523 \mathrm{~cm}^{-1}$ ). This is in good agreement with the literature [36].

Figure $3 \mathrm{~A}$ shows the SEM images of the uncoated Ti6AI4V screw-shaped dental implant and PLA biocomposite-coated Ti6Al4V screw-shaped dental implant (Fig. 3b) [37, 38]. While the hydroxyapatite microspheres are $100 \mathrm{~nm}$, the final coating is around $400 \mathrm{~nm}$ thick.

Drug release profiles of gentamicin-loaded PLA films and PLAHAp composites are shown in Fig. 4. It is possible

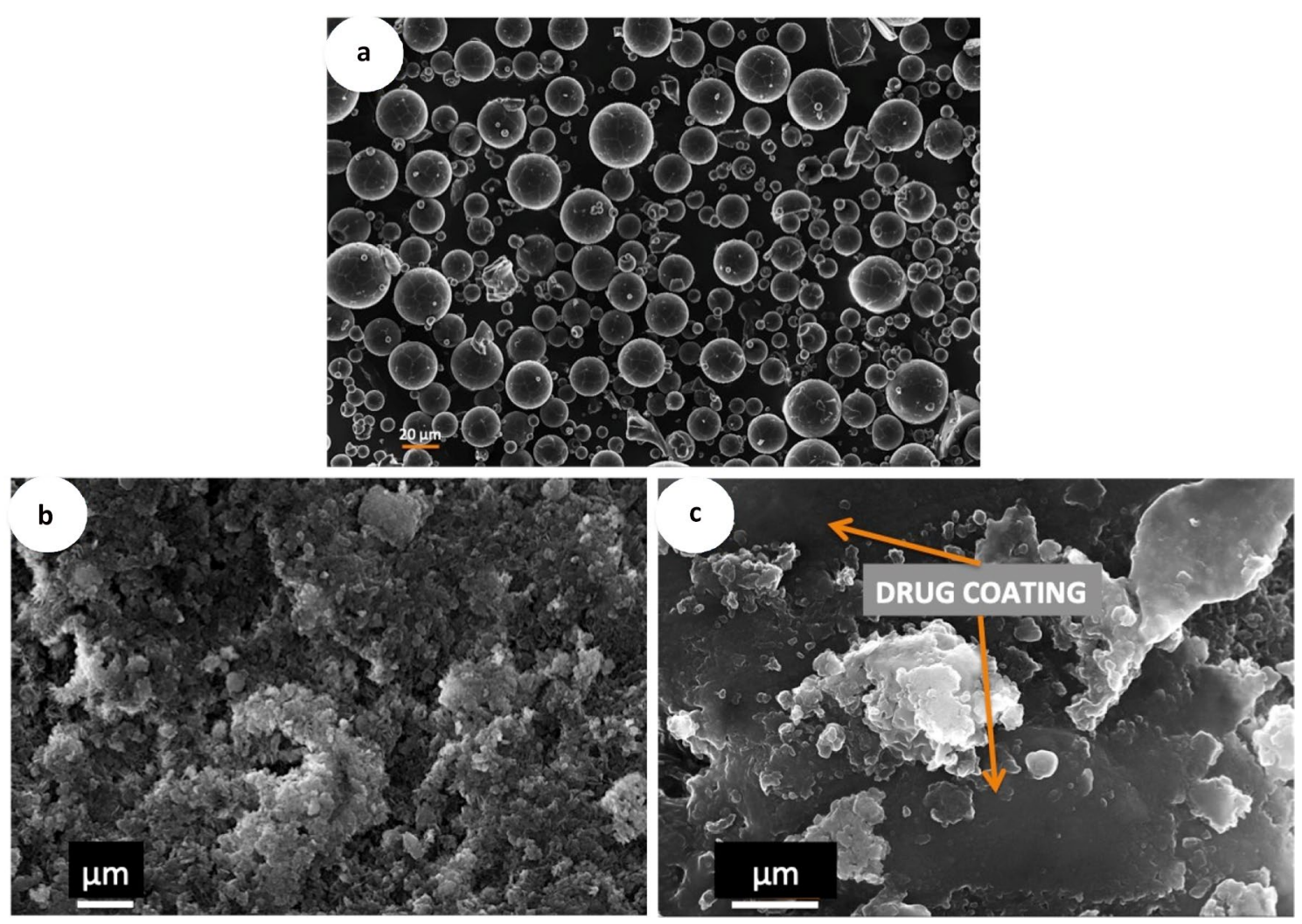

Fig. 1 a Gentamicin powder (spherical particles), b surface of HAp microspheres before drug loading, c Gentamicin-loaded HAp (coated with GM solution) 

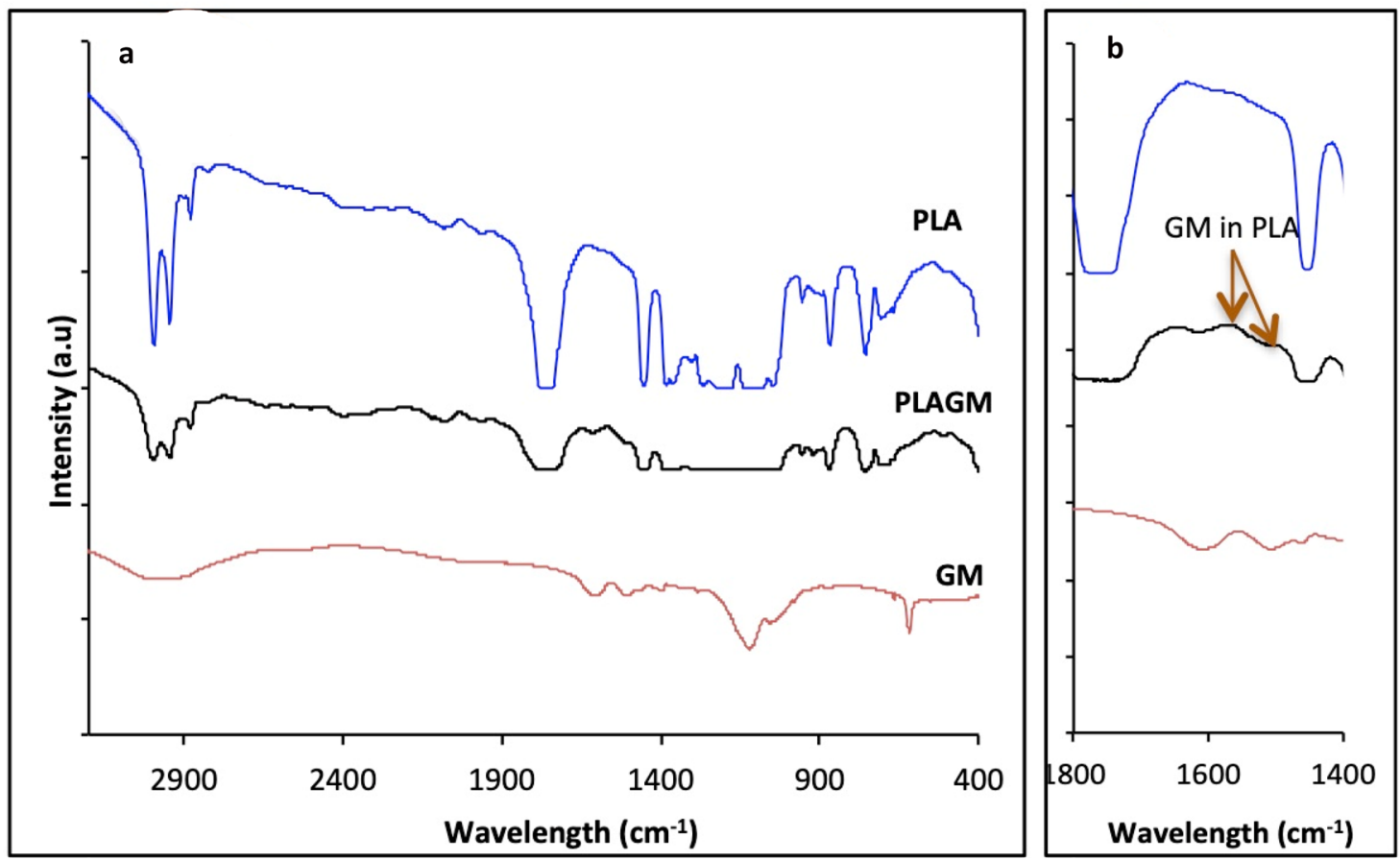

Fig. 2 FTIR spectra of drug-loaded PLA confirming non-denaturation of the drug in the PLA matrix, a after loading with Gentamicin, b showing the peaks due to gentamicin
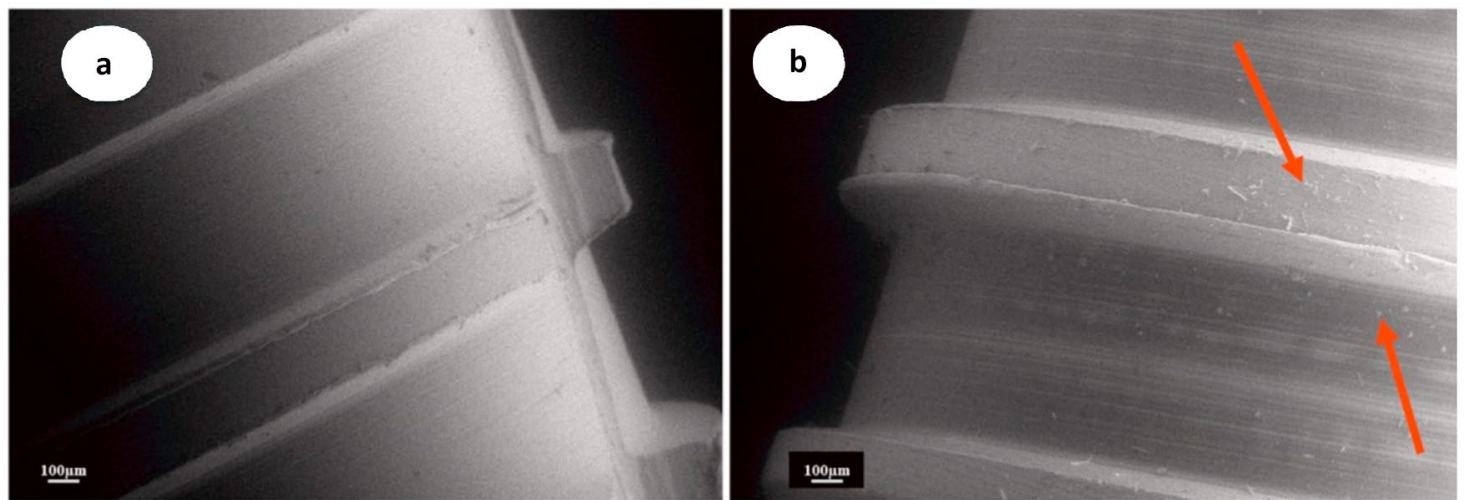

Fig. 3 a Uncoated dental implant surface, b PLAHApGm biocomposite thin-film-coated Ti6AL4V screw-shaped dental implant

that the first stage consists only of the surface-bound drug release assimilated as the direct dissolution of drug particles into water or solution. This process is relatively fast from few hours to a week ( $t=0-1$ week) and known as "burst effect". The second stage includes a number of sub-stages dependent on the drug particles and the environment and can be best summarized as "progressive cumulative dissolution" (PCD) ( $t=$ Week $1-5)$. In this stage, the polymer matrix starts to degrade and allows the liquid medium to penetrate into the HAp particles where HAp surface-bound drug attached to meso- and nanopores starts to dissolve and diffuse out. The rate of dissolution is slower due to the time required for the drug to diffuse through the HAp particles-matrix interface.

In the third or last stage ( $t=$ week $5-15)$ in addition to $P C D$, the particulate matter starts to break down (a relatively slow process) while the release rate is further reduced, but it includes the polymeric matrix degradation, HAp particle dissolution and further drug release at a steady-state rate.

This is known as the terminal release where degradation influences the drug release, which may surpass and prolong the drug diffusion mechanism. In our previous study [35], we showed that the release from these devices significantly prevented bacterial growth. The difference in 
Fig. 4 Fractional drug release from PLAHApGM and PLAGM films. Error bars are mean standard deviation (SD) of three experimental data

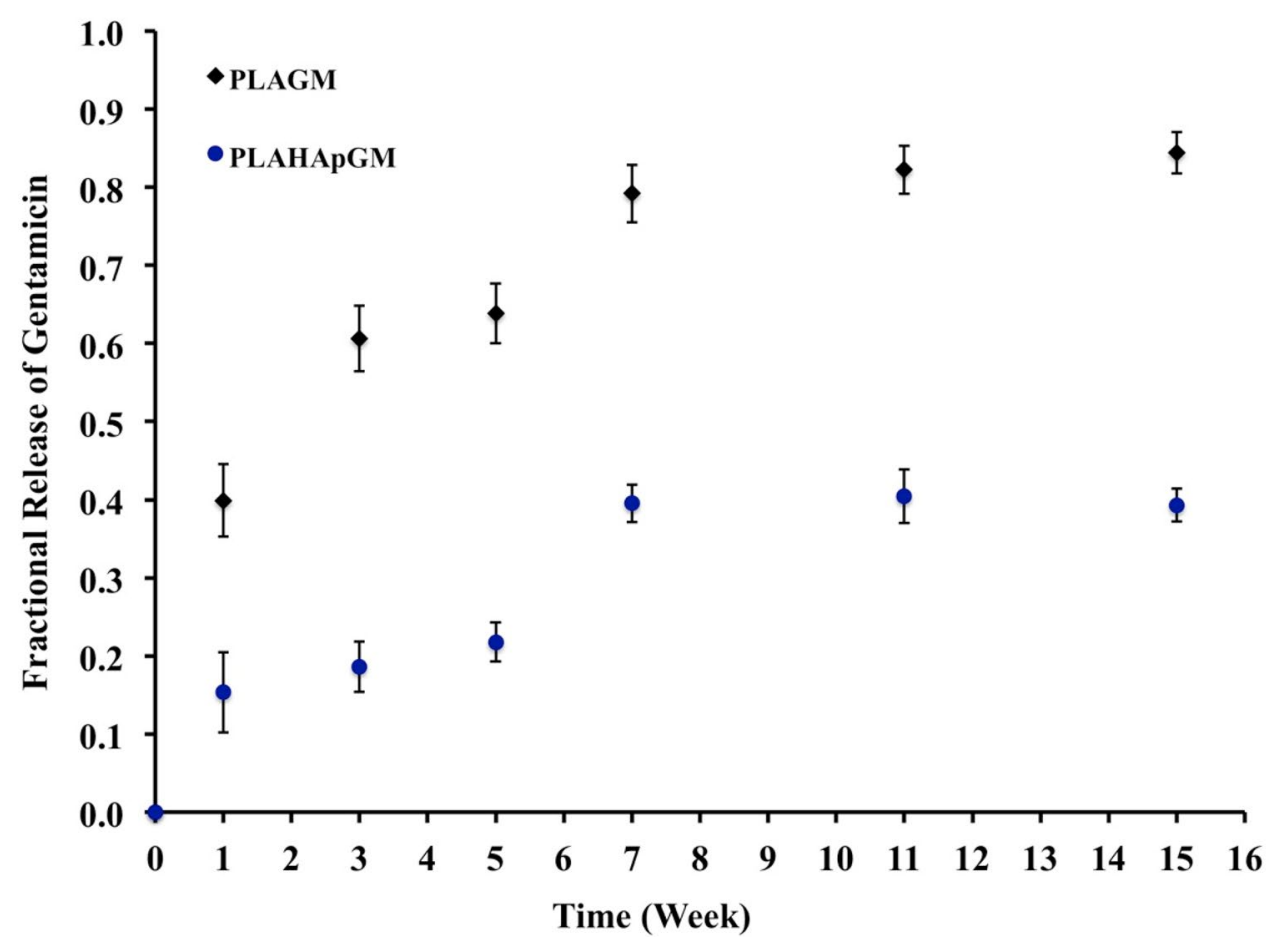

drug release between PLAGM and PLAHApGM is significant and can be assimilated by the fact that drug takes a long time to diffuse from HAp particles pores and then through PLA-HAP interface into the dissolution media. Having HAp particles embedded into the polymer matrix shows the potentials in materials combinational approach in controlled release of therapeutic agents.

Therefore, the release mechanism is mainly by diffusion from the polymer matrix influenced by the rate of larger pore formation, which principally is governed by polymer degradation rate. Different factors influence the degradation rate of the polymer matrix including polymer molecular weight and surface chemistry.

Four biofilm features, surface to bio-volume ratio, roughness coefficient, average thickness and biomass, were chosen and calculated by COMSTAT to characterize biofilm from $S$. aureus and P. aeruginosa on PLA thin-film composites. These variables were used to interpret biological and physical characteristics of biofilms based on previously published work [28]. The overall volume of biofilm is represented by biomass, while spatial biofilm size is represented by an average biofilm thickness. Biofilm heterogeneity is assimilated by roughness coefficient, while the surface to bio-volume ratio gives us information on the size of biofilm that was exposed to the nutrient flow. Figure 5 shows the variations of the biomass of $S$. aureus and $P$. aeruginosa on the surfaces of PLA composites films.

Figure 6 shows the average biofilm thickness on the surfaces of thin-film composites. The results are consistent with the biofilm volume shown in Fig. 5. P.aeruginosa showed characteristics

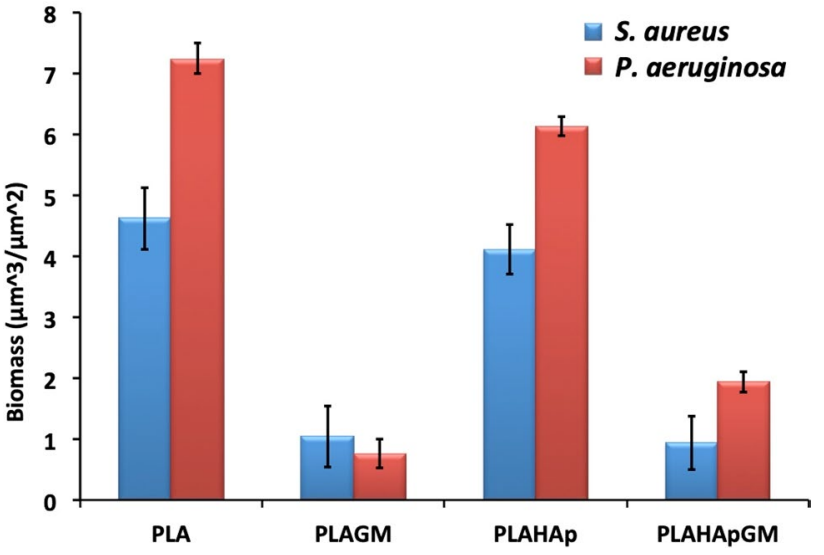

Fig. 5 5-day-old static grown biofilm of $S$. aureus and $P$. aeruginosa on PLA, PLAGM, PLAHAp, and PLAHApGM showing an average of biofilm volume (biomass), reveals relative large biomass for $P$. aeruginosa on PLA, PLAHAp, and PLAHAPGM and significant decrease in biomass for films loaded with gentamicin (PLAGM and PLAHApGM). The displayed error bars are mean standard deviation (SD) of triplicate experimental data

that are consistent with biomass and biofilm thickness due to its relatively high level of antimicrobial resistance.

\section{Conclusions}

The bacterial strains used in this study play a significant role in the medical context. The activities of these organisms have resulted in a growing interest in their 


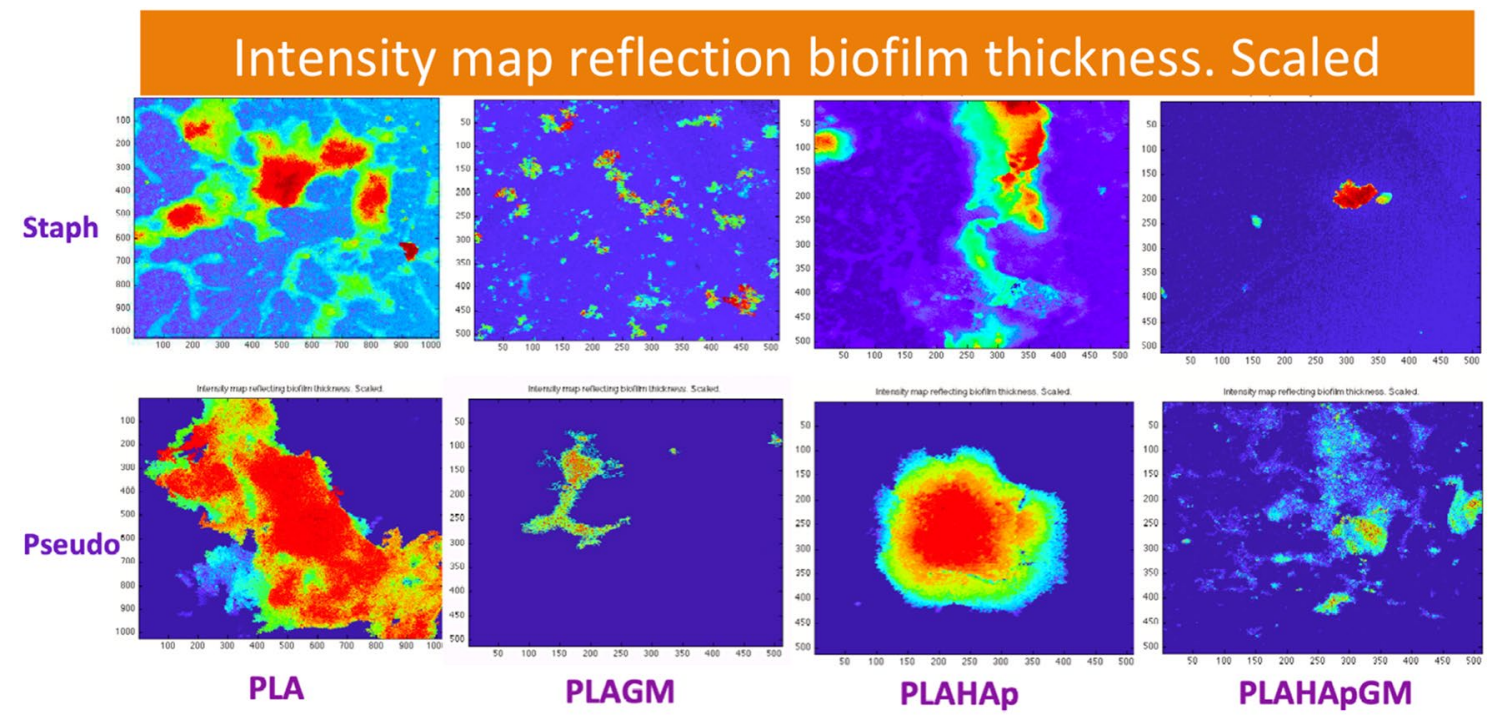

Fig. 6 5-day-old static grown biofilm of S. aureus and P. aeruginosa showing intensity mapping reflecting average biofilm thickness on PLA, PLAGM, PLAHAp and PLAHAPGM surfaces revealing large thickness for $P$. aeruginosa on PLA, PLAHAp and PLAHAPGM and a significant decrease in biofilm thickness for films loaded with gentamicin (PLAGM and PLAHApGM)

\section{Compliance with ethical standards}

cially in biofilm formations. It appears that biofilm development occurs differently on the same surfaces under different strains. Reduction in medical infections in dental, and orthopaedic implants and neural prostheses, lies in the ability to develop medical devices with modified materials. The release from these devices suggests that they can be used to release the clinical active substances in a prolonged period of time and could also be used to deliver the drugs in dental and orthopaedic implants and CNS and for the prevention of bacterial infections to neural prostheses. These implants can be coated by either spray or dip-coating techniques. Currently, the PLAHApGM coating has been tested on orthopaedic, dental implants and miniature 3D-printed devices and the results are promising. The release rate and degradation are all tuneable to suit specific applications. It is envisaged that control of hydroxyapatite particle sizes and their chemical composition and possible liposome coatings of the particles based on our previous work could influence the dissolution rates further. Further studies are highly needed to widen the current understanding of the biofilms and their prevention mechanisms in order to achieve the minimum number of medical implant and neuroprosthetics-related infection incidents.

Acknowledgements We are grateful for the financial support from the Australian Academy of Science and the European Union's Horizon 2020 research and innovation programme under the Marie Skłodowska-Curie Grant Agreement No 645749.
Conflicts of interest The authors declare no conflict of interest.

\section{References}

1. Kumar A, Pillai J (2018) Chapter 13: implantable drug delivery systems-an overview. In: Grumezescu AM (ed) Nanostructures for the engineering of cells, tissues and organs. William Andrew Publishing, New York, pp 473-511. https://doi.org/10.1016/ B978-0-12-813665-2.00013-2

2. Gavasane AJ, Pawar HA (2014) Synthetic biodegradable polymers used in controlled drug delivery system: an overview. Clin Pharmacol Biopharm 3(2):1-7. https://doi.org/10.4172/2167065X.1000121

3. Ben-Nissan B (2015) Discovery and development of marine biomaterials. In: Kim S-K (ed) Functional marine biomaterials, vol 100, 1st edn. Elsevier, Cambridge, pp 3-28

4. Macha IJ, Cazalbou S, Shimmon R, Ben-Nissan B, Milthorpe B (2017) Development and dissolution studies of bisphosphonate (clodronate)-containing hydroxyapatite-polylactic acid biocomposites for slow drug delivery. Journal of Tissue Engineering and Regenerative Medicine 11(6):1723-1731. https:// doi.org/10.1002/term.2066

5. Macha IJ, Charvillat C, Cazalbou S, Grossin D, Boonyang U, BenNissan B (2016) Comparative study of coral conversion, Part 3: intermediate products in the first half an hour. J Aust Ceram Soc 52(1):177-182

6. Macha IJ, Ozyegin LS, Chou J, Samur R, Oktar FN, Ben-Nissan B (2013) An Alternative Synthesis Method for Di Calcium Phosphate (Monetite) Powders from Mediterranean Mussel (Mytilus galloprovincialis) Shells. J Aust Ceram Soc 49(2):122-128

7. Dhar S, Gu FX, Langer R, Farokhzad OC, Lippard SJ (2008) Targeted delivery of cisplatin to prostate cancer cells by aptamer functionalized Pt(IV) prodrug-PLGA-PEG nanoparticles. Proc Natl 
Acad Sci USA 105(45):17356-17361. https://doi.org/10.1073/ pnas.0809154105

8. Griffith LG (2000) Polymeric biomaterials. Acta Mater 48(1):263277. https://doi.org/10.1016/S1359-6454(99)00299-2

9. Lou X, Detrembleur C, Jérôme R (2003) Novel Aliphatic Polyesters Based on Functional Cyclic (Di)Esters. Macromol Rapid Commun 24(2):161-172. https://doi.org/10.1002/marc.20039 0029

10. da Silva D, Kaduri M, Poley M, Adir O, Krinsky N, Shainsky-Roitman J, Schroeder A (2018) Biocompatibility, biodegradation and excretion of polylactic acid (PLA) in medical implants and theranostic systems. Chem Eng J 340:9-14. https://doi.org/10.1016/j. cej.2018.01.010

11. Thavornyutikarn B, Chantarapanich N, Sitthiseripratip K, Thouas GA, Chen Q (2014) Bone tissue engineering scaffolding: computer-aided scaffolding techniques. Progress in Biomaterials 3(2):61-102. https://doi.org/10.1007/s40204-014-0026-7

12. Tjong SC (2006) Structural and mechanical properties of polymer nanocomposites. Materials Science and Engineering: R: Reports 53(3):73-197. https://doi.org/10.1016/j. mser.2006.06.001

13. Thomas $P$ (2014) Clinical and diagnostic challenges of metal implant allergy using the example of orthopaedic surgical implants: part 15 of the Series Molecular Allergology. Allergo Journal International 23(6):179-185. https://doi.org/10.1007/ s40629-014-0023-3

14. Le VQ, Pourroy G, Cochis A, Rimondini L, Abdel-Fattah WI, Mohammed HI, Carrado A (2014) Alternative technique for calcium phosphate coating on titanium alloy implants. Biomatter 4:e28534. https://doi.org/10.4161/biom.28534

15. Ben-Nissan B, Macha I, Cazalbou S, Choi AH (2016) Calcium phosphate nanocoatings and nanocomposites, part 2: thin films for slow drug delivery and osteomyelitis. Nanomedicine. https ://doi.org/10.2217/nnm.15.220

16. Kinoshita M (2006) Targeted drug delivery to the brain using focused ultrasound. Topics in magnetic resonance imaging: TMRI 17(3):209-215. https://doi.org/10.1097/RMR.0b013e3180 $332 \mathrm{e} 79$

17. Kuittinen $O$, Siniluoto $T$, Isokangas $M$, Turpeenniemi-Hujanen T, Peltonen J, Alahuhta S, Sonkajarvi E (2013) Chemotherapy in conjunction with blood brain barrier disruption in the treatment of primary central nervous system lymphoma. Duodecim; laaketieteellinen aikakauskirja 129(15):1563-1570

18. Patel T, Zhou J, Piepmeier JM, Saltzman WM (2012) Polymeric Nanoparticles for Drug Delivery to the Central Nervous System. Adv Drug Deliv Rev 64(7):701-705. https://doi.org/10.1016/j. addr.2011.12.006

19. WHO (2007) Neurological disorders: public health challenges. WHO Press, Geneva

20. Yang $\mathrm{H}$ (2010) Nanoparticle-mediated brain-specific drug delivery, imaging, and diagnosis. Pharm Res 27(9):1759-1771. https ://doi.org/10.1007/s11095-010-0141-7

21. Roney C, Kulkarni P, Arora V, Antich P, Bonte F, Wu A, Mallikarjuana NN, Manohar S, Liang H-F, Kulkarni AR, Sung H-W, Sairam M, Aminabhavi TM (2005) Targeted nanoparticles for drug delivery through the blood-brain barrier for Alzheimer's disease. J Controll Release 108(2):193-214. https://doi.org/10.1016/j.jconr el.2005.07.024

22. Wilson B, Samanta MK, Santhi K, Kumar KPS, Ramasamy M, Suresh B (2010) Chitosan nanoparticles as a new delivery system for the anti-Alzheimer drug tacrine. Nanomed Nanotechnol Biol Med 6(1):144-152. https://doi.org/10.1016/j.nano.2009.04.001

23. Saraiva $C$, Praça $C$, Ferreira $R$, Santos $T$, Ferreira $L$, Bernardino $L$ (2016) Nanoparticle-mediated brain drug delivery: overcoming blood-brain barrier to treat neurodegenerative diseases.
J Controll Release 235:34-47. https://doi.org/10.1016/j.jconr el.2016.05.044

24. Ben-Nissan B (2003) Natural bioceramics: from coral to bone and beyond. Curr Opin Solid State Mater Sci 7(4):283-288. https:// doi.org/10.1016/j.cossms.2003.10.001

25. Ben-Nissan B, Green DW (2014) Marine structures as templates for biomaterials. In: Ben-Nissan B (ed) Advances in calcium phosphate biomaterials. Springer, Berlin, pp 391-414. https:// doi.org/10.1007/978-3-642-53980-0_13

26. Aviv M, Berdicevsky I, Zilberman M (2007) Gentamicin-loaded bioresorbable films for prevention of bacterial infections associated with orthopedic implants. J Biomed Mater Res Part A 83A(1):10-19. https://doi.org/10.1002/jbm.a.31184

27. Chou J, Valenzuela S, Green DW, Kohan L, Milthorpe B, Otsuka $M$, Ben-Nissan B (2014) Antibiotic delivery potential of nanoand micro-porous marine structure-derived beta-tricalcium phosphate spheres for medical applications. Nanomedicine 9(8):1131-1139. https://doi.org/10.2217/nnm.13.116

28. Heydorn A, Nielsen AT, Hentzer M, Sternberg C, Givskov M, Ersboll BK, Molin S (2000) Quantification of biofilm structures by the novel computer program COMSTAT. Microbiology $146(\mathrm{Pt}$ 10):2395-2407. https://doi.org/10.1099/00221287-146-10-2395

29. Kreth J, Hagerman E, Tam K, Merritt J, Wong DTW, Wu BM, Myung NV, Shi W, Qi F (2004) Quantitative analyses of Streptococcus mutans biofilms with quartz crystal microbalance, microjet impingement and confocal microscopy. Biofilms 1(4):277-284. https://doi.org/10.1017/S1479050504001516

30. Kreth J, Hagerman E, Tam K, Merritt J, Wong DT, Wu BM, Myung NV, Shi W, Qi F (2004) Quantitative analyses of Streptococcus mutans biofilms with quartz crystal microbalance, microjet impingement and confocal microscopy. Biofilms 1(4):277-284. https://doi.org/10.1017/S1479050504001516

31. Siepmann J, Siepmann F (2011) Mathematical modeling of drug release from lipid dosage forms. Int J Pharm 418(1):42-53. https ://doi.org/10.1016/j.ijpharm.2011.07.015

32. Rothstein SN, Federspiel WJ, Little SR (2008) A simple model framework for the prediction of controlled release from bulk eroding polymer matrices. J Mater Chem 18(16):1873-1880. https://doi.org/10.1039/B718277E

33. Rothstein SN, Federspiel WJ, Little SR (2009) A unified mathematical model for the prediction of controlled release from surface and bulk eroding polymer matrices. Biomaterials 30(8):16571664. https://doi.org/10.1016/j.biomaterials.2008.12.002

34. Stephens D, Li L, Robinson D, Chen S, Chang H-C, Liu RM, Tian Y, Ginsburg EJ, Gao X, Stultz T (2000) Investigation of the in vitro release of gentamicin from a polyanhydride matrix. $J$ Controll Release 63(3):305-317. https://doi.org/10.1016/S0168 -3659(99)00205-9

35. Fredenberg S, Reslow M, Axelsson A (2005) Measurement of protein diffusion through poly( $\mathrm{D}, \mathrm{L}$-lactide-Co-glycolide). Pharm Dev Technol 10(2):299-307. https://doi.org/10.1081/pdt-54473

36. Sionkowska A, Kaczmarek B, Gadzala-Kopciuch R (2016) Gentamicin release from chitosan and collagen composites. J Drug Deliv Sci Technol 35:353-359. https://doi.org/10.1016/j.jddst .2016.09.001

37. Macha IJ, Cazalbou S, Ben-Nissan B, Harvey KL, Milthorpe B (2015) Marine structure derived calcium phosphate-polymer biocomposites for local antibiotic delivery. Mar drugs 13(1):666680. https://doi.org/10.3390/md13010666

38. Karacan I, Macha IJ, Choi G, Cazalbou S, Ben-Nissan B (2017) Antibiotic containing poly lactic acid/hydroxyapatite biocomposite coatings for dental implant applications. Key Eng Mater 758:120-125. https://doi.org/10.4028/www.scientific.net/ KEM.758.120 